\title{
La promoción de salud como materia del currículum educativo en el contexto catalán
}

Health promotion as a subject of the educational curriculum in the Catalan context

\author{
Raquel Ayuso Margañón in \\ raquelayusom@ub.edu \\ Universidad de Barcelona. España
}

M. Cruz Molina Garúz

cmolina@ub.edu

Universidad de Barcelona. España

José Luis Medina Moya iD

jlmedina@ub.edu

Universidad de Barcelona. España

\section{Resumen}

OBjeTIVO. Comprender la manera en que se ha integrado la salud en el currículum educativo catalán y si ésta ha sido reconocida como una competencia más, contribuyendo con ello al logro de los objetivos de la nueva política europea para la promoción de la salud y el bienestar, denominada "Salud 2020". Reflexionar acerca del papel pedagógico del profesional de enfermería y proponer alternativas de mejora a la situación actual. MATERIALES Y MÉTODO. Se llevó a cabo un ejercicio interpretativo intencional y contextual articulándose el proceso en varias etapas: delimitación de las áreas de conocimiento curriculares a analizar en las etapas de escolarización obligatoria, redactado de los resultados y análisis de los datos surgidos. Como método analítico se utilizó el denominado análisis de contenido. Resultados. En la etapa de Primaria se observan competencias relacionadas con la salud en los ámbitos de conocimiento del medio; educación física; educación en valores y ámbito digital. En la etapa de Secundaria se observan competencias relacionadas con la salud en los ámbitos cientificotecnológico; social; educación física; cultura y valores; ámbito digital. CONCLUSIONES. la promoción de la salud no se proyecta como una materia más en la que se imparten contenidos de salud, sino como un conjunto de contenidos incluidos transversalmente en algunas de las áreas de conocimiento, lo que supondría una barrera para el conveniente desarrollo de los contenidos de salud. Se propone una nueva materia de promoción de salud, con contenidos adaptados a cada etapa educativa. El profesional de enfermería se erige como idóneo para adquirir la responsabilidad y liderazgo y poner en marcha dicha materia.

Palabras clave: salud escolar; planificación educativa; educación sanitaria; problemas de salud.

\begin{abstract}
OBJECTIVE. Understand how health has been integrated into the Catalan educational curriculum and whether it has been recognised as an additional competence, thereby contributing to the achievement of the objectives of the new European policy for the promotion of health and well-being, called "Health 2020". To reflect on the pedagogical role of the nursing professional and to propose alternatives for improvement to the current situation. MATERIALS AND METHODS. An intentional and contextual interpretative process was carried out, articulating the process in several stages: delimitation of the areas of curricular knowledge to be analyzed in the stages of compulsory schooling, writing up the results and data analysis. Content analysis was used as the analytical method. RESULTS. In the Primary Education stage, health-related competencies are observed in the fields of environmental knowledge, physical education, education in moral values and the digital field. At the Secondary level, competencies related to health are observed in the scientific-technological, social, physical education, culture and moral values and digital fields. CONCLUSIONS. Health promotion is not projected as just another subject in which health content is taught, but as a set of contents included transversally in some of the areas of knowledge, which would represent a barrier to the convenient development of health content. A new health promotion subject is proposed, with content adapted to each stage of education. The nursing professional becomes the most suitable choice to acquire responsibility and leadership and to implement this subject.
\end{abstract}

Keywords: school health; educational planning; health education; health problems.

Recibido / Received: 16-05-2018

Aceptado / Accepted: 10-09-2018

Cómo referenciar este artículo / How to reference this article:

Ayuso Margañón, R., Molina Garuz, M-C., \& Medina Moya, J.L. (2019). La promoción de salud como materia del currículum educativo en el contexto catalán. Tendencias Pedagógicas, 33, 83-98. doi: $10.15366 / \mathrm{tp} 2019.33 .007$ 


\section{Introducción}

El currículo educativo es el documento base que guía las actividades escolares para cada una de las etapas de enseñanza del sistema educativo en consonancia con la legislación vigente. Los currículos están orientados a la adquisición de las competencias que permiten desarrollar no sólo habilidades académicas, sino también personales y sociales que contribuyen al crecimiento de los alumnos y su incorporación a la sociedad. En otras palabras, el currículo encarna los objetivos y propósitos educativos de una sociedad (Stabback, 2016). Dichas competencias tienen su origen en la Recomendación 2006/962/EC, del Parlamento Europeo y del Consejo, de 18 de diciembre de 2006, sobre las competencias clave para el aprendizaje permanente, donde se aludía a la necesidad de la adquisición de "competencias clave" por parte de la ciudadanía, como condición indispensable para lograr la plena realización personal, la ciudadanía activa, la cohesión social y la empleabilidad en la sociedad del conocimiento (DOUE, 2006). A su vez, se destacaba la necesidad de medios para desplegar estas competencias durante la educación y la formación inicial. Se identificaban claramente ocho competencias clave esenciales para el bienestar de las sociedades europeas, el crecimiento económico y la innovación, y se describían los conocimientos, las capacidades y las actitudes esenciales vinculadas a cada una de ellas. En España se incorporaron al sistema educativo no universitario con el nombre de "competencias básicas" (comunicación lingüística, competencia matemática y competencias básicas en ciencia y tecnología; competencia digital; aprender a aprender; competencias sociales y cívicas; sentido de iniciativa y espíritu emprendedor; conciencia y expresiones culturales), siendo la Ley Orgánica 2/2006, de 3 de mayo, de Educación, conocida como LOE, la que introduce el término por primera vez en la normativa educativa (BOE, 2006). A partir de aquí, los Decretos por los que se establecen las enseñanzas mínimas de las diferentes etapas educativas incorporan estas competencias básicas al currículo, con objeto de enfatizar aquellos aprendizajes que se consideran imprescindibles para cada período educativo. Con la Ley Orgánica 8/2013, de 9 de diciembre, para la mejora de la calidad educativa (LOMCE), se dio un paso más al poner énfasis en un modelo basado en la potenciación del aprendizaje por competencias, integradas en los elementos curriculares (BOE, 2013).

En Cataluña, el currículum está fundamentado especialmente en los preceptos de la Ley 12/2009, de 10 de julio, de Educación (LEC), en las directrices de la Unión Europea, el Estatuto de autonomía de Cataluña, y en el Plan para la reducción del fracaso escolar en Cataluña 2012-2018, y pretende, entre otras cosas, consolidar el trabajo por competencias en la escuela (Gencat Dept Ensenyament, 2015). Teniendo en cuenta que la escuela ha sido reconocida por diferentes organismos como un entorno idóneo para la promoción de salud, cabe plantearse de qué manera se ha integrado la salud en el currículum educativo catalán y si ésta ha sido reconocida como una competencia más contribuyendo con ello al logro de los objetivos de la nueva política europea para la promoción de la salud y el bienestar, denominada "Salud 2020", como ya se viene haciendo en otros países de la Unión Europea. Organizaciones como UNESCO, UNICEF, Consejo de Europa, Comisión Europea, así como la Organización para la Cooperación y el Desarrollo Económicos (OCDE) avalan la promoción de salud en la escuela, considerando que se trata del modo más efectivo para promover la adopción y mantenimiento de estilos de vida saludables y el único camino para que la educación en salud llegue a los grupos de población más joven (Salvador, Suelves, \& Puigdollers, 2008).

Desde esta perspectiva, se plantea como objetivo en este estudio analizar si desde el ámbito educativo catalán se otorga la importancia merecida a la promoción de salud como para garantizar el crecimiento y el desarrollo no sólo individual, sino de toda la población. Asimismo, se proponen alternativas de mejora a la situación actual planteando el diseño de una materia curricular enfocada hacia la promoción de salud, y por último, se plantea el papel del profesional de enfermería en la implementación de dicha materia. Dicho estudio fue realizado en el contexto de una tesis doctoral.

\section{Material y métodos}

El análisis se centró en las etapas de enseñanza básica obligatoria (Primaria y Secundaria), dado que es a este alumnado, a los que la promoción de la salud debería llegar en mayor medida, 
esencialmente por la condición de obligatoriedad de la escolarización. Como fuente primaria se recurrió a la normativa legal correspondiente a los documentos curriculares para ambas etapas: Decreto 119/2015, de 23 de junio, de ordenación de las enseñanzas de la educación primaria (DOGC, 2015) y Decreto $187 / 2015$, de 25 de agosto, de ordenación de las enseñanzas de la educación secundaria obligatoria (DOGC, 2015b). Para completar el análisis fue necesaria la búsqueda en fuentes relacionadas estrechamente con el problema de estudio, extraídas de bases de datos con publicaciones de alto índice de impacto para las disciplinas de salud y educación. Igualmente, no se desestimaron otras fuentes tales como blogs científicos y otros documentos que constituyeron un valor importante para el estudio.

Se llevó a cabo un ejercicio interpretativo intencional y contextual articulándose el proceso en varias etapas: en primer lugar, se delimitaron las áreas de conocimiento a analizar (Tabla 1). Cada una de estas áreas —que conjuntamente al ámbito de conocimiento estructuran el currículum cataláncontribuye al desarrollo de las competencias básicas, que a su vez determinan el logro de los objetivos educativos de cada etapa. Después se delimitaron los hallazgos detectados y se realizó un proceso de relación y comparación de los datos, procediendo al redactado de los resultados. El análisis fue de carácter cualitativo y mayoritariamente inductivo de los datos surgidos en torno a los siguientes criterios: elementos curriculares relacionados con la salud dentro de las competencias básicas definidas y contenidos relacionados con la salud en las diferentes áreas curriculares. Como método se utilizó el denominado análisis de contenido. Este procedimiento ha sido considerado como apropiado para la descripción objetiva, sistémica y cualitativa del contenido manifiesto o implícito de una fuente de datos que el investigador debe interpretar (Krippendorff, 1991).

Tabla 1

Organización del curriculum educativo catalán para la etapa de Primaria y Secundaria

\begin{tabular}{|c|c|c|}
\hline & Ámbito & Áreas de conocimiento \\
\hline \multirow[t]{6}{*}{$\begin{array}{l}\text { Etapa de } \\
\text { Primaria }\end{array}$} & $\begin{array}{l}\text { Ámbito de } \\
\text { conocimiento y del } \\
\text { medio }\end{array}$ & - Conocimiento del medio natural, social y cultural \\
\hline & Ámbito artístico & - Educación artística (visual, plástica, música y danza) \\
\hline & $\begin{array}{l}\text { Ámbito de educación } \\
\text { física }\end{array}$ & - Educación física \\
\hline & $\begin{array}{l}\text { Ámbito lingüístico } \\
\text { Ámbito matemático }\end{array}$ & $\begin{array}{l}\text { - Lengua castellana/catalana y literatura } \\
\text { - Primera lengua extranjera } \\
\text { - Lengua aranesa (en la comarca de Arán). } \\
\text { - Matemáticas }\end{array}$ \\
\hline & $\begin{array}{l}\text { Ámbito de educación en } \\
\text { valores }\end{array}$ & $\begin{array}{l}\text { - Educación en valores sociales y cívicos o religión (en función } \\
\text { de la elección de las familias) }\end{array}$ \\
\hline & Ámbito digital & Transversal a todas las áreas \\
\hline \multirow[t]{2}{*}{$\begin{array}{l}\text { Etapa de } \\
\text { Secundaria }\end{array}$} & Ámbito Lingüístico & $\begin{array}{l}\text { - Lengua castellana/catalana y literatura } \\
\text { - Lenguas extranjeras } \\
\text { - Lengua aranesa (en la comarca de Arán) } \\
\text { - Latín (materia optativa) }\end{array}$ \\
\hline & Ámbito matemáticas & $\begin{array}{l}\text { - Matemáticas } \\
\text { - Matemáticas académicas aplicadas }\end{array}$ \\
\hline
\end{tabular}




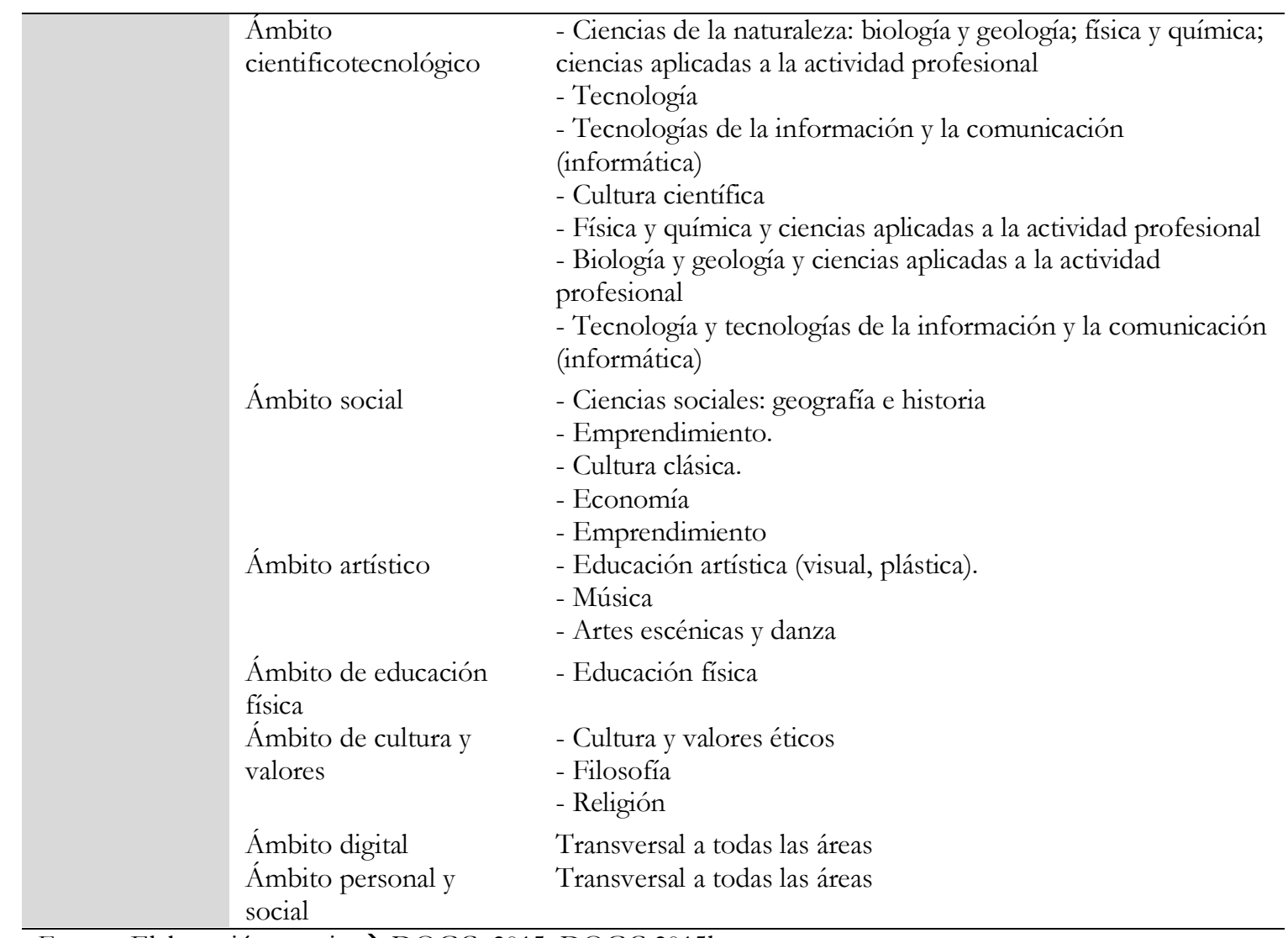

Fuente: Elaboración propia $\rightarrow$ DOGC, 2015; DOGC 2015b

\section{Resultados}

Se presentan a continuación los hallazgos observados para las etapas de escolarización obligatoria. Para la etapa de Primaria, los resultados están basados en la documentación correspondiente al Decreto 119/2015, de 23 de junio, de ordenación de las enseñanzas de educación primaria. En la etapa de secundaria, los resultados están basados en el Decreto 187/2015, de 25 de agosto, de ordenación de las enseñanzas de la educación secundaria obligatoria.

\subsection{Etapa de Primaria}

Se observan competencias relacionadas con la salud en los ámbitos de conocimiento del medio; educación física; educación en valores y ámbito digital.

- Ámbito de conocimiento del medio. Áreas de conocimiento del medio natural y de conocimiento del medio social y cultural. Las competencias básicas en este ámbito se agrupan en cuatro dimensiones, una de las cuales hace referencia a la salud. Se trata de la dimensión de salud y equilibrio personal, relacionada con el equilibrio físico, mental y social, y el logro de una vida autónoma, feliz y solidaria. Se podría afirmar que ésta es el área del currículum donde se incorpora la promoción de la salud de forma más explícita. Se reconoce la necesidad de disponer de unos recursos y conocimientos que permitan al alumno tomar decisiones oportunas de salud. Las competencias en esta dimensión están relacionadas con la adopción de hábitos sobre alimentación, actividad física y descanso para conseguir el bienestar. La toma de consciencia del propio cuerpo, de las emociones y sentimientos propios y ajenos para conseguir el equilibrio emocional y favorecer la convivencia. Y la toma de decisiones adecuadas sobre higiene y salud con conocimientos científicos para la prevención de enfermedades.

Los contenidos más específicos del área de conocimiento del medio natural y social en relación 
con las personas y la salud comprenden la identificación de las diferentes partes del cuerpo, nutrición y diferentes tipos de alimentos, principales enfermedades relacionadas con los órganos de los sentidos. La valoración de una alimentación sana y variada. Morfología extensa del cuerpo, hábitos de higiene, descanso, ejercicio que favorecen la vida saludable, expresión de sensaciones y emociones personales, respetando las del resto, gestión del tiempo libre, individual y colectivo en ciclo medio. Reconocimiento de la dimensión afectiva de la sexualidad y de otros órganos relacionados con la función de reproducción. Riesgos del consumo de tabaco y alcohol. Actuación de primeros auxilios para ayudarse y ayudar a los otros.

- Ámbito de educación física. Área de educación física. Este ámbito adquiere un carácter transversal, por tanto, establece relaciones con otras áreas dada su potencialidad educativa. Las competencias básicas también están agrupadas en cuatro dimensiones. Se resaltan dos de ellas en lo que se hace alusión a elementos curriculares de promoción de salud. Por un lado, la dimensión de actividad física, que reconoce la necesidad de conocer, desarrollar y fortalecer el cuerpo, mejorando el funcionamiento del aparato locomotor, cardiorrespiratorio, equilibrar el sistema nervioso, y proporcionar beneficios para la salud y el bienestar. Por otro lado, la dimensión de hábitos saludables, cuya competencia principal por adquirir es valorar la práctica habitual de la actividad física como factor beneficioso para la salud. Asimismo, como contenidos clave en relación con la actividad física y la salud, destaca el contenido de hábitos higiénicos, corporales y alimentación saludable.

- Ambito digital. Se parte de la base de que las competencias digitales son indispensables para toda persona que quiera interactuar con normalidad en la sociedad actual. Sus competencias se agrupan en cuatro dimensiones. Entre éstas, destaca por sus aportes en promoción de la salud la dimensión de hábitos, civismo e identidad digital, cuya competencia a subrayar en relación con la salud física y mental es desarrollar hábitos de uso saludable de la tecnología y actuar de forma crítica, prudente y responsable con las TIC. Se pretende que el alumno establezca la distinción entre la bondad que supone el buen uso de la tecnología e Internet y los perjuicios que le puede suponer adquirir conductas adictivas.

- Ámbito de educación en valores. Educación en valores sociales y cívicos. El objetivo es dar elementos a los alumnos para que sean capaces de vivir plenamente de manera responsable y feliz de tal forma que puedan contribuir a su vez al bienestar de quien los envuelve. El enfoque del área que lo compone ha de poner énfasis especial en el logro de actitudes y hábitos de conducta a lo largo de toda la etapa y en la adquisición de habilidades mentales autoconocimiento, de autocontrol, de empatía y de asertividad. Las dimensiones que lo estructuran en las que se hallan aportes de promoción de salud están especialmente en el contexto social, afectivo y emocional. Por un lado, la dimensión personal, que incluye las competencias que prevén el reconocimiento de las emociones y sentimientos propios y las habilidades de pensamiento y de razonamiento que han de orientar la capacidad de tomar decisiones y actuar de manera autónoma y crítica. Además, habilidades para hacer frente a los cambios y dificultades para alcanzar el bienestar personal. Por otro lado, la dimensión interpersonal, que pretende preparar al alumnado para una relación harmónica y respetuosa con los otros y para la resolución de las situaciones de conflicto a través del diálogo. Y, por último, la dimensión social, que comporta las competencias de analizar el entorno con criterios éticos y buscar soluciones alternativas a problemas y mostrar actitudes de servicio y compromiso social.

En lo que respecta a los contenidos clave a destacar, están los relacionados con habilidades sociales, tales como sentimientos y principios morales, identificación de emociones propias y de los demás, empatía, asertividad, actitudes que contribuyen al bienestar emocional del grupo, hábitos cívicos y de convivencia.

\subsection{Etapa de Secundaria}

En la etapa de Secundaria se observan competencias relacionadas con la salud en los ámbitos cientificotecnológico, social, educación física, cultura y valores, ámbito digital. A continuación, se 
muestran los aportes encontrados en las áreas que lo componen.

- Ámbito cientificotecnológico. El despliegue de las competencias se agrupa en cuatro dimensiones. De éstas, se pueden encontrar aspectos relacionadas con la promoción de salud en una de ellas: la dimensión salud, relacionada con la capacitación para adoptar medidas de prevención y hábitos saludables a nivel individual y social, fundamentadas en el conocimiento de las estrategias de detección y respuesta del cuerpo humano. Y la capacitación para aplicar medidas preventivas en relación con las conductas de riesgo y enfermedades asociadas al consumo de sustancias adictivas. Adoptar hábitos de alimentación variada y equilibrada con objeto de evitar trastornos alimentarios y enfermedades asociadas. Y dar respuesta a las cuestiones sobre sexualidad y reproducción humanas, valorando las consecuencias de las conductas de riesgo.

En lo que respecta a los contenidos clave relacionados con la promoción de salud, destacan aquellos relacionados con el efecto de las conductas de riesgo y de los condicionantes sociales sobre la salud individual y colectiva. Prevención y buenos hábitos. Efectos psíquicos de las drogas sobre el organismo, factores de riesgo y de protección del consumo de drogas y consecuencias sociales del consumo. Tolerancia, dependencia física y psicológica. Y enfermedades asociadas a la nutrición.

- Ámbito social. Estructurada en cuatro dimensiones: histórica, geográfica, cultural y artística, ciudadana. La que aborda elementos de promoción de salud es esta última. La consecución de las competencias que incluye conlleva respetar la identidad propia y la de los demás, rechazando comportamientos y actitudes irracionales, desarrollando valores, habilidades y actitudes fundamentales de convivencia, con una atención especial a la discriminación de género.

Los contenidos clave más afines a la promoción de salud en este ámbito están relacionados con la pertinencia y cohesión social. Situación de desigualdad, discriminación, aportes que pueden responder a una buena salud emocional y social.

- Ámbito de educación física. Las competencias básicas para este ámbito se despliegan en cuatro dimensiones, destacando por sus aportaciones en promoción de salud la dimensión de actividad física saludable. Por un lado, está dirigida a la práctica de actividad física para la mejora o mantenimiento de la condición física y, por otro lado, dirigida a la adquisición del conocimiento y valoración de los efectos beneficiosos y perjudiciales para la salud derivados de los diferentes estilos de vida.

Algunos de los contenidos clave son afines a la higiene corporal y la salud física y psicológica en la práctica de actividades físicas. Hidratación, alimentación, descanso. Salud y sustancias tóxicas. Respiración y relajación. El cuerpo como modelo de identidad social.

- Ámbito cultura y valores. Estructurada en tres dimensiones, incluye entre otras, competencias dirigidas a mejorar la autonomía personal del alumnado fundamentada en la deliberación reflexiva, serena y con criterios, considerando las consecuencias de los propios actos y los haga conscientes de las responsabilidades que se derivan de ello. Las competencias más afines a la promoción de salud, especialmente en el contexto emocional y mental para este ámbito, se reparten entre la dimensión personal, relacionadas con la autonomía en la toma de decisiones y responsabilidad en los propios actos. Y la dimensión interpersonal, relacionadas con las actitudes de respeto hacia los demás y aplicación de diálogo en la resolución de conflictos.

En cuanto a contenidos clave relacionados con la promoción de la salud destacan los fundamentos en la toma de decisiones (decisiones en salud, por ejemplo). Los derechos humanos como deberes morales (por ejemplo, en la violencia de género), situaciones y contextos de conculcaciones de los derechos humanos y de las libertades. Valores universales y valores compartidos. Actitudes de tolerancia. Microagresiones y bullying.

- Ámbito digital. En lo que se refiere a la salud, encontramos la relación en las conductas de uso y abuso de las tecnologías. Se trata de fomentar una actitud crítica en esta área para contribuir a la prevención de problemas de conducta y adicciones. Así como el conocimiento de las posibles 
lesiones físicas y psíquicas derivadas de un trabajo continuado con objeto de prevenir riesgos. Además, gestión eficaz de la identidad digital, la privacidad y reputación personal, que contribuye a la salud mental y social. De las cuatro dimensiones, la que aporta estas competencias relacionadas con la salud es la dimensión ciudadana, hábitos, civismo e identidad digital.

Los contenidos educativos a destacar vinculados a la promoción de salud: ergonomía, salud física y psíquica. Identidad digital y riesgos de una mala gestión de la privacidad.

\section{Discusión}

Atendiendo a los resultados obtenidos, se puede afirmar que la promoción de la salud no se proyecta como una materia más en la que se imparten contenidos de salud, sino como un conjunto de contenidos incluidos transversalmente en algunas de las áreas de conocimiento. Además, si comparamos el currículum educativo vigente de Cataluña con el anterior de $2007^{9}$ cimentado en los preceptos de la LOE, se observa que no ha habido cambios significativos en lo que se refiere a los contenidos de salud para las etapas de Primaria y Secundaria. Si bien es cierto que la LEC fue pionera en dejar manifiesta la necesidad de abordar problemas de salud emergentes de la realidad social, como es el caso de la violencia de género y acoso escolar; y que el vigente currículum educativo de Cataluña se ha intentado adaptar a los cambios, añadiendo en los contenidos educativos aportes de promoción de la salud sobre conductas de riesgo presentes en la sociedad actual (bullying, uso de las tecnologías digitales, entre otros), sin embargo, en lo que se refiere a su puesta en marcha, no ha habido cambios relevantes a lo largo de estos últimos años. Materias tradicionales como matemáticas o geografía han tenido más empuje en la educación que otros aspectos también importantes como el ambiente emocional y social (Palacio, \& Sáez, 2001), y la promoción de la salud se sigue proyectando de forma puntual en las áreas de conocimiento, lo que supondría una barrera para el conveniente desarrollo de los contenidos de salud. Estos hallazgos son concordantes con otros autores quienes consideran que los proyectos de promoción de la salud generalmente no se incluyen en el currículum, no se trabaja la salud del propio ambiente escolar y, aunque las intervenciones se extienden de alguna manera a la familia y a la comunidad educativa, se desarrollan fundamentalmente en el aula (Davó, Gil-González, Vives-Cases, Álvarez-Dardet, \& La Parra, 2008). Por otro lado, se apunta a la falta de participación, la falta de coordinación entre escuela, la familia y el ámbito sanitario, así como los programas curriculares sobrecargados (Talavera \& Gavidia, 2013).

La salud, en tanto que es corpus de conocimiento consolidado y en expansión, más que una materia transversal, debería ser abordada sistemáticamente, adaptada a los diversos niveles de desarrollo de los alumnos, como una materia específica y obligatoria dentro del currículum formativo escolar y no sólo tratarse de manera trasversal (García, Sáez, \& Escarbajal, 2000; Monsalve, 2013). Cuando los programas se acortan de manera significativa, se excluyen actividades relativas a alguna conducta, y cuando se llevan a cabo en ámbitos distintos a los previstos, los resultados positivos originales no se reproducen (Jané et al., 2013). La mayor parte de los programas que han tenido efectos perdurables en el comportamiento consisten en sesiones secuenciales de dos o tres años de duración, o bien, en programas donde la mayoría de las sesiones se realizan durante el primer año y son seguidas por sesiones de reforzamiento y/o profundización (UNESCO, 2010).

Por otro lado, al no ser de carácter obligatorio, y quedar relegada a la transversalidad, las intervenciones de salud no llegan a todo el alumnado de igual manera, quedando subordinada a la responsabilidad y compromiso del maestro/a, el cual muchas veces lo afronta con falta de recursos y de formación en esta temática que obstaculizan su abordaje (Monsalve, 2013). De hecho, entre las cinco grandes barreras detectadas por la OMS para la implementación de la promoción de salud en la escuela destaca la escasa formación de maestros/as y personal de la escuela en los conceptos generales de los programas de salud y pocas escuelas tienen las habilidades para ponerlas en práctica

\footnotetext{
${ }^{9}$ Decreto 142/2007, de 26 de junio, por el que se establece la ordenación de las enseñanzas de la educación primaria y Decreto 143/2007, de 26 de junio, por el que se establece la ordenación de las enseñanzas de la educación secundaria obligatoria.
}

Tendencias Pedagógicas, 33, 2019, pp. 83-98. DOI: 10.15366/tp2019.33.007 
(WHO, 1997). En el Informe de la Unión Internacional de Promoción de la Salud y Educación para la Salud para la Comisión Europea se llegaba a similares conclusiones (IUEP, 2000). Acercándonos al contexto catalán, en un estudio donde se exploró el papel del profesorado en la educación para la salud en la escuela en la ciudad de Gerona, se confirmaba que más del 50\% del profesorado consideraba tener poca preparación, y únicamente el 20\% afirmaba haber tratado aspectos relacionados con la salud como actividad complementaria de alguna asignatura a lo largo de su formación universitaria. Este mismo estudio ratificaba que la mayoría del profesorado no tiene claro su papel ejemplar, pudiendo transmitir patrones o modelos de conducta no saludables y entrar en contradicción entre lo que dicen en clase y lo que ocurre fuera del aula, al no ser conscientes de que pueden ser imitados (Juvinyà, 2008).

Desde esta perspectiva, se ha evidenciado el papel pedagógico en la salud del profesional de enfermería. Allí donde se ha instituido, ha contribuido a conseguir una mejor salud para la población escolar. En el Healthy People $2010^{10}$ se reconoció que están en una posición ideal para promover y proporcionar educación para la salud y reducir los riesgos de los estudiantes (Baisch, Lundeen, \& Murphy, 2011). En un estudio llevado a cabo de 2006-2009 en el que se evaluaba la eficacia de la intervención enfermera en las escuelas para dejar de fumar, se demostró que dicha intervención resultó factible y eficaz en la mejora de la abstinencia a corto plazo entre los adolescentes varones. Éstos eran casi dos veces más propensos a la abstinencia a los 3 meses de la intervención, en comparación con el grupo control, consiguiendo unas tasas de abandono del 15\% de los adolescentes, frente al 4,9\% del grupo control. Asimismo, cantidad y frecuencia de fumadores, disminuyó significativamente en ambos sexos a los 3 meses (Pbert et. al., 2011). En un estudio en que se evaluaba un programa de intervención educativa realizado por un profesional de enfermería dirigido a fortalecer los conocimientos en salud bucal en los escolares, se demostró que el nivel de conocimiento de los escolares en relación con este tema mejoró respecto del que tenían previamente a la realización de la intervención, pasando del 21\% al 64\% (Rivera, Maldonado, Castro, \& Balderas, 2011).

En una investigación sobre el papel del enfermero en la escuela y su impacto en reducir al mínimo las desigualdades en salud en la absorción de la vacuna del virus del papiloma humano (VPH), Boyce \& Holmes (2012) demostraron que la intervención del profesional de enfermería escolar aumentó la captación de niñas susceptibles de ser inmunizadas, incluso aquellas de difícil acceso; determinando que este profesional juega un papel clave en la reducción de las desigualdades en salud, no sólo en el programa de VPH, sino también en otros programas de vacunación de la infancia y la adolescencia; ya que mejoran la comprensión de los procesos de entrega de dichos programas, y pueden aportar información y soluciones para aumentar la absorción y hacer frente a las desigualdades en este tema. Asimismo, en un estudio que evaluaba la viabilidad y la eficacia de la intervención enfermera integrada en la escuela, en lo que se refiere a la mejora de la dieta, la actividad física y reducción del índice de masa corporal (IMC) entre los adolescentes con sobrepeso y obesidad; se determinó que mejoraron los comportamientos relacionados con la obesidad de quienes participaron (Pbert et. al., 2013).

La UE sigue apostando por la promoción de salud como parte importante de la estrategia de crecimiento y desarrollo hacia una "Europa 2020" (DOUE L/86, 2014). Asimismo, las nuevas normativas educativas dejan claro que a través del nuevo currículo se pretende abrir una puerta a este enfoque, sin embargo, en lo que respecta al ámbito educativo, el presupuesto en España es cuestionable, siendo uno de los países que menos invierte en educación. Según el último informe, en 2014, los países de la OCDE (Organización para la Cooperación y el Desarrollo Económico) dedicaron de media un 5,2\% de su PIB a instituciones educativas; en España este porcentaje fue del 4,3\%, uno de los más bajos de toda la UE y en Cataluña, aún menor, 3,60\% (IDESCAT, 2015; MECD, 2015). Se hace imprescindible, por tanto, invertir en normativas específicas que garanticen

\footnotetext{
${ }^{10}$ Declaración de los objetivos nacionales de salud en EEUU, diseñados para identificar las amenazas prevenibles más importantes para la salud y para establecer objetivos nacionales con objeto de reducir dichas amenazas. Proporciona un marco de referencia en salud. Actualmente está siendo desarrollada la Healthy People 2020. Extraído de la web oficial http://www.healthypeople.gov/2010/
} 
una formación en salud de calidad en la escuela. Existe suficiente conocimiento científico para poder asegurar que un alto nivel de salud mejora la productividad en todos los ámbitos analizados, de ahí que la inversión en salud, incluyendo la inversión en las estructuras que aseguran la salud de la población (como es la escuela), sea una de las políticas públicas más rentables y, si además respeta la equidad efectiva, esencialmente justa (BOE, 2011).

Es el momento de considerar la concepción de una nueva materia de promoción de la salud, con contenidos de salud adaptados a cada etapa educativa a lo largo de todo el curso escolar, cuya configuración abarque competencias de salud que permitan un desarrollo integral y óptimo de la persona y una alfabetización científica que permita desarrollar hábitos saludables a largo plazo. Esto significaría, que la mayoría de la población disponga de los conocimientos científicos y tecnológicos necesarios para desenvolverse en la vida diaria, ayudar a resolver los problemas y necesidades de salud y supervivencia básicos, tomar conciencia de las complejas relaciones entre ciencia y sociedad y, en definitiva, considerar la ciencia como parte de la cultura de nuestro tiempo (Furió \& Vilches, 1997). Por tanto, el principal objetivo de dicha materia es desarrollar actuaciones de forma continuada que favorezcan la adquisición de competencias en salud; conocimientos, habilidades y actitudes para el desarrollo positivo y para afrontar las situaciones de riesgo. Se trata en definitiva de acciones que faciliten al alumnado la elección y adopción de estilos de vida saludable. La responsabilización y empoderamiento del individuo en su propia salud, que a su vez lleva a la adquisición de hábitos saludables perdurables. Esto se puede conseguir cuando el alumno es capaz de entender y tomar conciencia de las consecuencias de sus conductas de riesgo para la salud, en un contexto educativo en el que incluso el alumno puede estar aprendiendo sin ser completamente consciente del proceso. (Cope \& Kalantzis, 2009).

Así, partiendo de las recomendaciones de protocolos y guías para garantizar un desarrollo saludable en la etapa infantil-juvenil, así como de otros referentes que también avalan la educación socioeducativa y ambiental en la escuela se propone una selección de contenidos repartidos en cuatro dimensiones: física, mental, afectiva-sexual-reproductiva, y social, que podrían conformar esta nueva materia en relación con las necesidades del currículo educativo catalán, adaptando las intervenciones a los diferentes ciclos (Tabla 2).

Tabla 2

Estructura y contenido de la materia curricular propuesta: Promoción de salud

Dimensión Descripción Contenidos




\begin{tabular}{|c|c|c|}
\hline $\begin{array}{l}\text { Dimensión } \\
\text { física }\end{array}$ & $\begin{array}{l}\text { Competencias relacionadas con } \\
\text { el desarrollo de hábitos } \\
\text { favorables a la salud física y } \\
\text { autocuidado en situación de } \\
\text { enfermedad. Incluye la } \\
\text { responsabilización de la propia } \\
\text { salud y la de los demás; } \\
\text { conductas de riesgo, problemas } \\
\text { asociados a éstas y sus } \\
\text { consecuencias en la salud física; } \\
\text { gestión de situaciones de riesgo } \\
\text { a través de medidas } \\
\text { preventivas. }\end{array}$ & $\begin{array}{l}\text { Las intervenciones para trabajar los contenidos que } \\
\text { se describen a continuación, deben de estar } \\
\text { adaptadas a las diferentes etapas evolutivas: } \\
\text { - Hábitos alimentarios. Problemas de desarrollo } \\
\text { físico relacionados con anomalías del peso y la } \\
\text { alimentación: obesidad motivada por exceso de } \\
\text { ingesta calórica y/o costumbres de la familia; } \\
\text { retraso ponderal motivado por trastornos de la } \\
\text { conducta alimentaria (anorexia, bulimia, } \\
\text { intolerancias y alergias alimentarias, entre otros). } \\
\text { Propiedades alimentarias. Alimentación equilibrada, } \\
\text { control de grasas. } \\
\text { - Ejercicio físico. Inactividad física y sedentarismo. } \\
\text { Prevención de problemas a largo plazo } \\
\text { (cardiovasculares, dolor articular y contracturas, } \\
\text { bajo estado de ánimo, autoestima baja, entre otros). } \\
\text { Educación postural (sedestación, ordenador, } \\
\text { transporte de material escolar, entre otros). } \\
\text { - Hábitos tóxicos y consumo de drogas. Prevención } \\
\text { de problemas relacionados con los efectos nocivos } \\
\text { del alcohol, tabaco y otras sustancias psicoactivas. } \\
\text { Hábitos familiares. } \\
\text { - Higiene y salud bucodental (caries, enfermedad } \\
\text { periodontal, maloclusiones). Higiene diaria y acné. } \\
\text { Higiene del sueño. } \\
\text { - Seguridad y prevención de accidentes. Accidentes } \\
\text { domésticos, de tráfico y en el deporte. Primeros } \\
\text { auxilios. Vacunación y sistema inmunológico } \\
\text { (desmitificación y familiarización). } \\
\text { - Riesgos medioambientales para la salud y el } \\
\text { bienestar, contaminación del aire y el agua, } \\
\text { contaminación acústica y productos químicos } \\
\text { tóxicos. }\end{array}$ \\
\hline
\end{tabular}




\begin{tabular}{|c|c|c|}
\hline $\begin{array}{l}\text { Dimensión } \\
\text { mental }\end{array}$ & $\begin{array}{l}\text { Teniendo en cuenta que la } \\
\text { escuela es el marco donde se } \\
\text { desarrollan los aprendizajes y } \\
\text { las capacidades cognitivas, de } \\
\text { sociabilidad y de relación, esta } \\
\text { dimensión es indispensable } \\
\text { para la adquisición de } \\
\text { competencias que permitan } \\
\text { potenciar y promover el } \\
\text { adecuado desarrollo psicológico } \\
\text { en la infancia y la adolescencia } \\
\text { a través de actividades } \\
\text { educativas preventivas de tipo } \\
\text { psicológico y psicosocial, en } \\
\text { función de las diferentes } \\
\text { necesidades y ritmos de } \\
\text { aprendizaje del alumnado. } \\
\text { Actitudes y habilidades de } \\
\text { respuesta y afrontamiento ante } \\
\text { determinadas situaciones } \\
\text { cotidianas, así como en } \\
\text { situaciones conflictivas, que } \\
\text { pueden conformar problemas } \\
\text { de aprendizaje, rendimiento } \\
\text { escolar y maduración personal. }\end{array}$ & $\begin{array}{l}\text { Los contenidos se resumen en: } \\
\text { - Autoestima, autoconcepto, autovaloración. } \\
\text { Aceptación y respeto de la propia realidad corporal } \\
\text { e identidad personal, y la de los demás. Relacionado } \\
\text { con trastornos de personalidad en la edad adulta. } \\
\text { - Abordaje de situaciones estresantes en la } \\
\text { infancia/adolescencia. Estrés asociado con la } \\
\text { escuela. Sobreestimulación de información. } \\
\text { Relacionado con síntomas de depresión, problemas } \\
\text { de atención, apatía, agresividad, entre otros. } \\
\text { - Gestión de emociones/inteligencia emocional. } \\
\text { Conocimiento y empleo de las emociones de forma } \\
\text { positiva. Manejo de sentimientos de tristeza, } \\
\text { irritabilidad, culpa, falta de ilusión, euforia, miedo. } \\
\text { Relacionado con trastornos del comportamiento } \\
\text { (desobediencia, mentira, baja tolerancia a la } \\
\text { frustración, entre otras). } \\
\text { - Relaciones familiares. Competencias para la } \\
\text { identificación y abordaje de ambiente anormal y } \\
\text { factores sociales estresantes. Manejo de } \\
\text { sentimientos negativos ante conflictos y dificultades } \\
\text { familiares. } \\
\text { - Conducta responsable en relación a la propia } \\
\text { salud. Autocuidado y cuidado de los demás. } \\
\text { Desarrollo de actitud reflexiva y crítica. Desarrollo } \\
\text { de la expresión de opiniones, sentimientos y } \\
\text { emociones en relación a la salud. Toma de } \\
\text { decisiones adecuadas de forma independiente. } \\
\text { - Falta de atención y/o falta de concentración. } \\
\text { Relacionado con trastornos por déficit de atención, } \\
\text { problemas de conducta social y fracaso escolar. } \\
\text { Habilidades de concentración y hábitos de estudio. } \\
\text { Manejo de la impulsividad. Trazar metas y } \\
\text { objetivos. }\end{array}$ \\
\hline
\end{tabular}




\begin{tabular}{|c|c|c|}
\hline $\begin{array}{l}\text { Dimensión } \\
\text { afectiva-sexual- } \\
\text { reproductiva }\end{array}$ & $\begin{array}{l}\text { A través de intervenciones } \\
\text { educativas en esta dimensión, } \\
\text { se trata de fomentar una visión } \\
\text { de la salud afectiva, sexual y } \\
\text { reproductiva desde una } \\
\text { perspectiva positiva, coherente } \\
\text { con los valores de la } \\
\text { comunidad, basada en la } \\
\text { responsabilidad, los derechos } \\
\text { sexuales, la equidad de género y } \\
\text { de los derechos sexuales. } \\
\text { Para ello, se han de potenciar } \\
\text { competencias reproductivas, } \\
\text { afectivas, relacionales y de } \\
\text { género, a través de } \\
\text { intervenciones de perspectiva } \\
\text { abierta, con enfoque global, sin } \\
\text { desvincular las conductas } \\
\text { sexuales de otros aspectos } \\
\text { personales y vivenciales. } \\
\text { Se trata de fomentar } \\
\text { habilidades para la vida, y no } \\
\text { únicamente centradas en la } \\
\text { teoría y la técnica. }\end{array}$ & 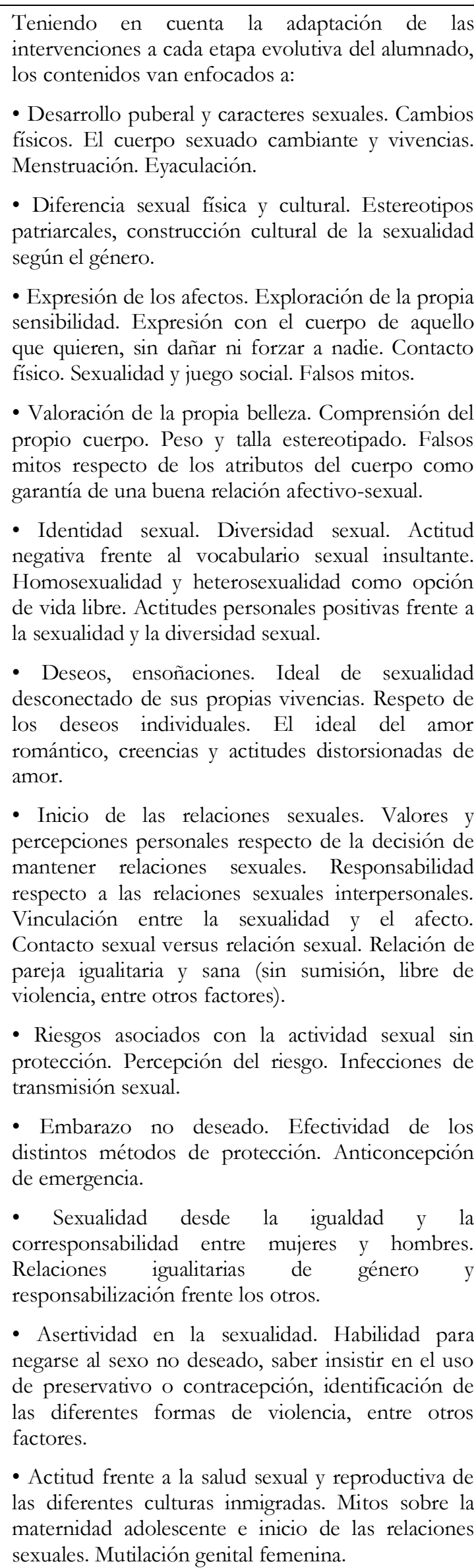 \\
\hline
\end{tabular}




\begin{tabular}{|c|c|c|}
\hline & & $\begin{array}{l}\text { - Competencias socioafectivas: autoestima y } \\
\text { autoconcepto. Valoración de actitudes sexuales } \\
\text { individuales, normas percibidas, autoeficacia. }\end{array}$ \\
\hline $\begin{array}{l}\text { Dimensión } \\
\text { social }\end{array}$ & $\begin{array}{l}\text { Se organiza en torno a la } \\
\text { interacción con otras personas } \\
\text { y a los factores del entorno. En } \\
\text { este sentido, se trata de } \\
\text { desarrollar competencias que } \\
\text { permitan entender la salud } \\
\text { contextualizada en las } \\
\text { diferentes situaciones sociales, } \\
\text { teniendo en cuenta los riesgos } \\
\text { del entorno y la conexión entre } \\
\text { los comportamientos } \\
\text { individuales y los estímulos } \\
\text { sociales. } \\
\text { Se trata de entender la salud } \\
\text { como una responsabilidad } \\
\text { individual y compartida. } \\
\text { Comporta la adquisición de } \\
\text { competencias relacionadas con } \\
\text { la propia identidad personal, la } \\
\text { adquisición de la idea de uno } \\
\text { mismo como base de } \\
\text { construcción del propio } \\
\text { proyecto vital, optando por } \\
\text { conductas saludables. } \\
\text { Desarrollo de valores } \\
\text { fundamentales de convivencia y } \\
\text { compromiso social, desechando } \\
\text { conductas irracionales que } \\
\text { comprometan la propia salud y } \\
\text { la de los demás. } \\
\text { Y, por último, capacidad de } \\
\text { analizar el entorno y los } \\
\text { factores que lo componen con } \\
\text { criterio de salud y buscar } \\
\text { soluciones alternativas a } \\
\text { problemas derivados de éste. }\end{array}$ & $\begin{array}{l}\text { Los contenidos deben estar encaminados a la } \\
\text { consecución de la buena salud del alumnado y del } \\
\text { centro escolar: } \\
\text { - Sentimiento de pertinencia y cohesión social. } \\
\text { Estrategias de resolución de conflictos. Pandillas. } \\
\text { - Redes sociales. Riesgo social digital. Principio } \\
\text { ético en la utilización de los medios digitales. } \\
\text { - Utilización de normas de convivencia pacífica y } \\
\text { tolerante } \\
\text { - Violencia entre iguales. Bullying. Aislamiento. } \\
\text { Inhibición social. } \\
\text { - Desigualdades. Violencia de género. Separación } \\
\text { entre niños y niñas. } \\
\text { - Actitud positiva hacia el medio para preservar la } \\
\text { salud. Clima escolar. }\end{array}$ \\
\hline
\end{tabular}

Fuente: Elaboración propia $\rightarrow$ European Comission, 2016; García, J. (coord), 2003 ; Graciela, H., \& Jaramillo, C., 2006; March M, \& Orte C. (coords), 2014; Prats, B., Prats, R., \& Plasència, A., (coords), 2008; Soler, E., \& del Campo, E., 2014; UNESCO, 2010.

\section{Conclusiones}

A través de este artículo se ha analizado la manera en que se ha integrado la promoción de salud en el currículum educativo y cómo ha contribuido esto en el cumplimiento de los objetivos poblacionales de salud para una nueva estrategia europea "Salud 2020". Como resultado se ha evidenciado que la transversalidad interfiere en la correcta alfabetización científica del tema de salud y adquiere cada vez más importancia la necesidad de asignar un tiempo considerable a la educación para la salud en el plan de estudios, integrando intervenciones de promoción de salud en la dinámica de la escuela, de forma continuada y permanente, desde la formación inicial o etapas iniciales de aprendizaje, quedando contemplado además en la legislación educativa. Asimismo, se ha evidenciado la necesidad de que dicha formación sea impartida por personal especializado y motivado para ello. Esto también contribuiría a la sistematización en la oferta de contenidos de salud y al aumento del sentimiento de pertenencia percibido y del compromiso desde el ámbito educativo con el cumplimiento de los objetivos de salud, al estar normalizada 
como una asignatura más del currículum educativo.

Los contenidos de salud planificados, e impartidos de forma continuada incorporados al currículum escolar, pueden contribuir a la adquisición de conocimientos, actitudes y habilidades que capacitan para el cuidado de la propia salud y la de la colectividad, así como al desarrollo de una conciencia crítica tanto en la identificación de riesgos de salud, como en la toma de decisiones. Pero todo ello implica una voluntad política, un incremento de recursos destinados a la planificación de intervenciones en este tema y la creación de un fuerte marco legal con una normativa que otorgue a la promoción de la salud una asignación suficiente en el plan de estudios que abarque todas las etapas educativas por igual, garantizando una educación para la salud firme, segura y eficaz.

Desde esta perspectiva, la propuesta de una materia de promoción de salud generada para este artículo tiene la ventaja de poseer contenidos adaptables a cada etapa educativa, cuya configuración abarca la dimensión física, psicológica-emocional, afectiva-sexual, y social del currículum, es decir, abarca competencias de salud repartidas en estas dimensiones, permitiendo así un desarrollo integral del educando. Dicha materia requeriría de una planificación adecuada y de asignación considerable en el plan de estudios para asegurar la continuidad en las intervenciones educativas en salud; así como las alianzas estratégicas entre los sectores saludeducación, y la impartición por parte de profesionales expertos en esta área, como es en este caso, el profesional de enfermería. Como limitación cabe destacar el contexto geográfico al que se ha limitado el artículo, no obstante, aunque la propuesta sigue los criterios del currículum catalán, también podría hacerse extensivo a otros contextos geográficos.

\section{Referencias}

Baisch, M. J., Lundeen, S. P., \& Murphy, M. K. (2011). Evidence-Based Research on the Value of School Nurses in an Urban School System. Journal of School Health, 81(2), pp. 74-80. doi: 10.1111/j.1746-1561.2010.00563.x

BOE. (2006). Ley Orgánica 2/2006, de 3 de mayo, de Educación (LOE). BOE 04-05-2006, núm. 106, pp. 17158-17207. Ref. BOE-A-2006-7899

BOE. (2011). Ley 33/2011, de 4 de octubre, General de Salud Pública (LGSP). Madrid: BOE 05-10-2011, núm. 240, pp. 104593-104626. Ref. BOE-A-2011-15623

BOE. (2013). Ley Orgánica 8/2013, de 9 de diciembre, para la mejora de la calidad educativa (LOMCE). BOE 30-12-2013, núm. 295, pp. 97858-97921. Ref. BOE-A-2013-12886

Boyce, T., \& Holmes, A. (2012). Addressing Health Inequalities in the Delivery of the Human Papillomavirus Vaccination Programme: Examining the Role of the School Nurse. PLoS ONE, 7(9), 1-7. doi: 10.1371/journal.pone.0043416

Cope, B. \& Kalantzis, M. (Eds.). 2009 Ubiquitous learning. Exploring the anywhere/anytime possibilities for learning in the age of digital media. University of Illinois Press, Recuperado el 17 de julio de 2018, de http://www.nodosele.com/blog/wpcontent/uploads/2010/03/Cope_Kalantzis.Aprendizajeubicuo.pdf

Davó, M. C., Gil-González, D., Vives-Cases, C., Álvarez-Dardet, C., \& La Parra, D. (2008). Las investigaciones sobre promoción y educación para la salud en las etapas de infantil y primaria de la escuela española. Una revisión de los estudios publicados entre 1995 y 2005. Gaceta Sanitaria, 22(1), pp. 58-64. doi:10.1157/13115112

DOGC. (2015). Decret 119/2015, de 23 de juny, d'ordenació dels ensenyaments de l'educació primària. Barcelona: DOGC 26/06/2015, núm 6900, pp. 1-139. Ref. CVE-DOGC-B-15176019-2015

DOGC. (2015b). Decret 187/2015, de 25 d'agost, d'ordenació dels ensenyaments de l'educació secundària obligatòria. Barcelona: DOGC 28/08/2015, núm 6945, pp. 1-305. Ref. CVE-DOGC-A15237051-2015

DOUE. (2006). Recomendación del Parlamento Europeo y del Consejo, de 18 de diciembre de 2006, sobre competencias clave para el aprendizaje permanente. DOUE L/394, 2006. pp. 10-8

DOUE. (2014). Reglamento (UE) núm. 282/2014 del Parlamento Europeo y del Consejo, de 11 de marzo de 2014, relativo a la creación de un tercer programa de acción de la Unión en el ámbito de la salud para el periodo 2014-2020 y por el que se deroga la Decisión núm. 1350/2007/CE. Estrasburgo: DOUE L/86/1-13, 2014. pp. $1-13$

European Comission. 2016. Environment. 7th EAP priority objectives. Recuperado el 12 de julio 2018, 
de http://ec.europa.eu/environment/action-programme/objectives.htm

Furió, C. \& Vilches, A. (1997). Las actitudes del alumnado hacia las ciencias y las relaciones ciencia, tecnología y sociedad, en Luis del Carmen (Coord.). La enseñanza y el aprendizaje de las ciencias de la naturaleza en la educación secundaria. Barcelona: Horsori.

García, A., Sáez, J., \& Escarbajal, A. (2000). Educación para la salud apuesta por la calidad de vida. Madrid: Arán Ediciones S.A.

García, J. (coord). (2003). Recomanacions per a l'atenció dels trastorns mentals greus en la infäncia $i$ l'adolescència: Quadern de salut mental 6 (1a ed.). Barcelona: Gencat. Dept. de Sanitat i Seguretat Social.

Gencat Dept Ensenyament. (2015). Un currículum competencial per a l'educació primària: avançament. Recuperado el 18 de junio de 2017, de http://xtec.gencat.cat/web/.content/alfresco/d/d/workspace/SpacesStore/0060/8756225aeeda-4b31-b9b3-f4df03e0de5e/Quadriptic_decret.pdf

Graciela, H., \& Jaramillo, C. (2006). La educación sexual de niñas y niños de 6 a 12 años: guía para madres, padres y profesorado de Educación Primaria. Madrid: Secretaría General Técnica. Centro de Publicaciones. Ministerio de Educacion y Ciencia. Recuperado 4 de junio de 2017, de: https://sede.educacion.gob.es/publiventa/la-educacion-sexual-de-ninas-y-ninos-de-6-a-12anos-guia-para-madres-padres-y-profesorado-de-educacion-primaria/educacion/12059

IDESCAT. (2015). Gasto público en educación respecto al PIB. Recuperado 2 de septiembre de 2017, de https: / $/$ www.idescat.cat/economia/inec?tc $=3 \&$ id $=8308 \& l a n g=e s$

IUHPE. (2000). The evidence of Health Promotion Effectiveness: Shaping Public Health in a New Europe. A report for the European Commission by the International Union for Health Promotion and Education (Part two. Evidence book). Recuperado el 12 de junio de 2017, de http://www.iuhpe.org/images/PUBLICATIONS/THEMATIC/EFFECTIVENESS/HPE_E vidence-1_EN.pdf

Jané, M., Amorós, P., \& Molina, M. C., Vidal, M. J., Mateu, A. (2013). Pla de salut afectiva i sexual (PSAS): Promoció i prevenció en la infància $i$ l'adolescència, amb especial èmfasi en la població vulnerable (1a ed.) [versión electrónica]. Barcelona: Agència de Salut Pública de Barcelona. Departament de Salut. Recuperado 3 de mayo de 2017, de http://hdl.handle.net/11351/1277

Juvinyà, D. (2008). L'educació per a la salut a l'escola $i$ el paper del professorat. (Tesis doctoral). Universidad de Gerona, Gerona. Recuperado 15 de mayo de 2017, de http://www.tdx.cat/handle/10803/7981

Krippendorff, K. (1991). Metodologia del análisis de contenido. Buenos Aires: Paidós-Comunicación.

March M, \& Orte, C. (coords). (2014). Los retos socioeducativos de la institución escolar en el siglo XXI. Barcelona: Octaedro.

MECD. (2015). Panorama de la educación. Indicadores de la OCDE 2015: Informe español. (2015). Madrid: Ministerio de Educación, Cultura y Deporte. Secretaría General Técnica. Recuperado 12 de septiembre de 2016, de http://www.mecd.gob.es/dctm/inee/internacional/panorama-de-laeducacion-2015.-informe-espanol.pdf?documentId=0901e72b81 ee9fa3

Monsalve, L. (2013). Politicas educativas en educación para la salud en la unión europea: Estudio comparado. (Tesis doctoral) Facultad de Filosofía y Ciencias de la Educación, Valencia. Recuperado de http://roderic.uv.es/bitstream/handle/10550/31150/Tesis Doctoral Laura Monsalve septiembre 2013.pdf?sequence $=1$

Palacio, M., \& Sáez, A. (2001). Entornos Saludables: el desafío de la Promoción de la Salud. En H. E. Restrepo \& H. Málaga (Eds.), Promoción de la Salud: cómo construir vida saludable (pp. 264-273). Bogotá: Editorial Médica Internacional LTDA

Pbert, L., Druker, S., DiFranza, J. R., Gorak, D., Reed, G., Magner, R., ..., \& Osganian, S. (2011). Effectiveness of a school nurse-delivered smoking-cessation intervention for adolescents. Pediatrics, 128(5), pp. 926-36. doi: 10.1542/peds.2011-0520

Pbert, L., Druker, S., Gapinski, M. A., Gellar, L., Magner, R., Reed, G., ..., \& Osganian, S. (2013). A School Nurse-Delivered Intervention for overweight and obese adolescents. Journal of School Health, 83(3), pp. 182-193. doi: 10.1111/josh.12014

Prats, B., Prats, R., \& Plasència, A., (coords). (2008). Protocol d'activitats preventives i de promoció de la salut a l'edat pediàtrica: infància amb salut (1 a ed.). Barcelona: Agència de Salut Pública de Catalunya. Recuperado 01 de junio de 2017, de http://hdl.handle.net/11351/1197

Rivera, P., Maldonado, G., Castro, R., \& Balderas, Y. (2011). Evaluación de Intervención Educativa 
de Enfermería en Salud Bucal en Niños. Desarrollo Cientifico de Enfermería, 19(5), pp. 175-178. Recuperado el 2 de junio de 2017, de http://www.index-f.com/dce/19pdf/19-175.pdf

Salvador, T., Suelves, J. M., \& Puigdollers, E. (2008). Guia para las administraciones educativas y sanitarias. Criterios de Calidad para el Desarrollo de Proyectos y Actuaciones de Promoción y Educación para la Salud en el Sistema Educativo. Madrid: Educación, Política Social y Deporte (Centro de Innovación y Documentación Educativa, CIDE) y Ministerio de Sanidad y Consumo (Dirección General de Salud Pública). Recuperado el 10 de agosto de 2017, de http://www.msssi.gob.es/profesionales/saludPublica/prevPromocion/promocion/saludjovene s/docs/criteriosCalidad2MSC_MEC.pdf

Soler, E., \& del Campo, E. (2014). La educación para la salud en la escuela: Una herramienta con poderes de cambios positivos en los hábitos saludables de la sociedad: Programa de Educación para la Salud en las Escuelas $e$ Institutos (PESEI). Recuperado 20 de junio de 2017, de: http://acise.cat/wpcontent/uploads/2015/09/PESEI-2014-2015-castellano-PDF.pdf

Stabback P. (2016). Qué hace a un curriculo de calidad. Reflexiones en progreso no 2 sobre cuestiones fundamentales y actuales del curriculo y el aprendizaje. Ginebra: UNESDOC. Recuperado el 15 de julio de 2017, de http://unesdoc.unesco.org/images/0024/002439/243975s.pdf

Talavera, M., \& Gavidia, V. (2013). Percepción de la educación para la salud en el personal docente y el sanitario. Didáctica de Las Ciencias Experimentales Y Sociales, O(27), pp. 115-129. doi: $10.7203 /$ dces. 27.2569

UNESCO. (2010). Orientaciones Técnicas Internacionales sobre Educación en Sexualidad. Un enfoque basado en evidencia orientado a escuelas, docentes y educadores de la salud (Volumen I: Justificación de la educación en sexualidad). París: UNESCO. Recuperado 2 de junio de 2017, de http://unesdoc.unesco.org/images/0018/001832/183281 s.pdf

WHO. (1997). Promoting Health Through Schools: Report of a WHO Expert Committee on Comprehensive School Health Education and Promotion (WHO Technical Report Series 870). Recuperado el 12 de julio de 2017, de http://apps.who.int/iris/bitstream/handle/10665/41987/WHO_TRS_870.pdf;jsessionid=92D 67ACE6AC5531A5FF67B5204FAF008? sequence $=1$ 